\title{
Análise comparativa entre a refração clínica subjetiva e a automatizada obtida por sensor de frentes de onda
}

\author{
Comparative analyses between clinical refraction and automatic refraction obtained \\ through a wave front sensor
}

\author{
Wilson de Freitas ${ }^{1}$ \\ Luiz Alberto Soares Melo Júnior \\ Paulo Schor ${ }^{3}$ \\ Mauro Campos ${ }^{4}$
}

Trabalho realizado no Setor de Cirurgia Refrativa do Departamento de Oftalmologia da Universidade Federal de São Paulo - UNIFESP - São Paulo (SP) - Brasil.

${ }^{1}$ Doutor em Medicina, Universidade Federal de São Paulo - UNIFESP - São Paulo (SP) - Brasil.

2 Pós-graduando, Universidade Federal de São Paulo UNIFESP - São Paulo (SP) - Brasil.

${ }^{3}$ Livre docente, Professor do Departamento de Oftalmologia da UNIFESP - São Paulo (SP) - Brasil.

${ }^{4}$ Livre docente, Professor do Departamento de Oftalmologia da UNIFESP - São Paulo (SP) - Brasil.

Endereço para correspondência: Wilson de Freitas. Rua Comendador Cantinho, 555 - São Paulo (SP)

CEP 03603-020 - E-mail: wfreitas@uol.com.br

Recebido para publicação em 23.05.2006

Última versão recebida em 13.03.2007

Aprovação em16.04.2007

Nota Editorial: Depois de concluída a análise do artigo sob sigilo editorial e com a anuência dos Drs. Breno Barth e Vinícius Coral Ghanem sobre a divulgação de seus nomes como revisores, agradecemos sua participação neste processo.

\section{RESUMO}

Objetivo: Avaliar e comparar os resultados obtidos da refração estática clínica com a obtida por sensor de frentes de onda. Métodos: Estudo prospectivo, não seqüencial, de 279 olhos de 147 pacientes. Todos os pacientes foram examinados sob cicloplegia. Primeiro realizamos a refração clínica e a seguir a automatizada por sensor de frentes de onda. Os dados refracionais obtidos foram decompostos para análise vetorial. Foram analisados separadamente os dados de um olho por paciente e dos dois olhos. Resultados: A diferença entre a refração clínica e a automatizada foi de $-0,19$ DE combinada com $-0,06$ DC no eixo de $15^{\circ}$ para os dados de um olho por paciente e $-0,17$ DE combinada com $-0,05$ DC no eixo de $3^{\circ}$ para todos os olhos da amostra. Conclusão: Os dados da refração clínica são comparáveis com os da refração obtida por um sensor de frentes de onda.

Descritores: Erros de refração/cirurgia; Refração ocular; Optometria; Técnicas de diagnóstico oftalmológico; Estudo comparativo

\section{INTRODUÇÃO}

A cirurgia refrativa vem atualmente passando por uma fase de avanço tecnológico, onde a busca por cirurgias reprodutíveis, seguras e principalmente pela individualização dos tratamentos, vem produzindo melhores resultados $^{(1)}$. Uma tecnologia que está sendo explorada pelos cirurgiões refrativos é o estudo das aberrações oculares pela análise de frentes de onda, que quando acoplado ao excimer laser proporciona um tratamento personalizado àquele olho. As aberrações oculares podem ser divididas em baixa e alta ordem, onde as de baixa ordem correspondem basicamente ao erro refrativo, miopia, hipermetropia e astigmatismo, enquanto que as de alta ordem avaliam distúrbios de magnitude menores que contribuem para diminuição da acuidade visual, seja em quantidade ou em qualidade ${ }^{(2)}$.

Como estes sistemas de análise por frentes de ondas para as cirurgias personalizadas oferecem o resultado da refração ocular, desenvolvemos este trabalho para avaliar a reprodutibilidade deste exame, comparando os resultados obtidos pela refração clínica com os obtidos pelo aparelho sensor de frentes de onda, ambos sob cicloplegia.

\section{MÉTODOS}

Este estudo prospectivo, não seqüencial, foi realizado com aprovação do Comitê de Ética em Pesquisa da Universidade Federal de São Paulo/ Hospital São Paulo. Foram analisados 279 olhos de 147 pacientes, sendo 67 
$(46,3 \%)$ do sexo masculino e $80(53,7 \%)$ do feminino, com média de idade de 32,1 $( \pm 7,9)$ anos, idade mínima e máxima de 21 e 53 anos. Estes pacientes procuraram o Serviço de Cirurgia Refrativa do Departamento de Oftalmologia da Universidade Federal de São Paulo (UNIFESP), por demanda espontânea, para submeterem-se à cirurgia corretiva de suas respectivas ametropias no período de março a julho de 2003.

Como critério de inclusão consideramos todos os pacientes portadores de uma ametropia, e que entendessem e colaborassem com os exames de refratometria realizados. Foram excluídos pacientes com: alterações da transparência do cristalino, alterações do filme lacrimal, astigmatismo irregular topográfico, ectasias da córnea, presença de deformidade na superfície da córnea induzida por utilização de lente de contato, afacia, pseudofacia, catarata, subluxação do cristalino e cirurgia ocular prévia.

Foi seguida a rotina do exame oftalmológico pré-operatório para pacientes com indicação à cirurgia refrativa:

1) Identificação, anamnese, ectoscopia, exames videoceratográficos por anel de Plácido (Eyesys Techonologies Version 3.04, Houston, TX, EUA) ou por varredura em fenda (Orbscan II, Bausch\&Lomb - Orbtek Inc, Salt Lake City, UT, EUA).

2) Refratometria clínica sob cicloplegia utilizando o refrator automático (Topcon KR 7000 - Topcon, Paramus, NJ, EUA) com a média de cinco medidas e por meio de recursos propedêuticos não automatizados incluindo retinoscopia, refrator tipo "Greens", cilindro cruzado de Jackson nos astigmatismos, teste bicromático para os míopes e determinação da melhor acuidade visual. A cicloplegia foi realizada com colírio de ciclopentolato $1 \%$, instilando-se uma gota em cada olho duas vezes, com intervalo de cinco minutos entre a instilação das gotas e o exame realizado após quarenta minutos. O resultado do poder dióptrico esférico e cilíndrico foi anotado em múltiplos de 0,25 dioptrias, o cilíndrico expresso no valor negativo e o eixo variando de 5 em 5 graus.

3) Seguindo a refratometria clínica, foi realizada a refratometria automatizada utilizando o sensor de frentes de onda (LadarWave ${ }^{\circledR}$ Custom Cornea Wavefront System - Alcon Laboratories Inc, Orlando, FLA, (USA), com cinco medidas em cada olho, para obtenção da média refracional. O componente esférico das medidas do sensor de frentes de onda abrangeu de $+8,00$ dioptrias a $-18,00$ dioptrias e o componente cilíndrico até 6,0 dioptrias, com posição de eixo de $0^{\circ}$ a $180^{\circ}$. Os resultados do poder dióptrico esférico e cilíndrico variou em 0,01 dioptrias, o valor do cilíndrico negativo e o eixo variando de 1 em 1 grau.

4) Os diagnósticos das ametropias foram dados pelos resultados refracionais e considerou-se o valor zero do componente cilíndrico para ametropias esféricas.

5) Exame biomicroscópico.

6) Mapeamento de retina.

7) Tonometria de aplanação tipo Goldman.

8) Paquimetria

As refrações clínica e automatizada foram agrupadas em valores esféricos, cilíndricos com seus eixos e classificadas quanto ao tipo de ametropia.

\section{Análise estatística}

Para a realização da análise matemática convertemos os valores esfero-cilíndricos da refração subjetiva clínica e da obtida por um sensor de frentes de onda para o formato polar de vetores de força (magnitude e direção) antes da realização dos cálculos.

Na conversão dos valores utilizamos o método de Naeser, que usa a equação $\mathrm{MV}(90)=\mathrm{m}\left(\operatorname{sen}^{2} \alpha-\cos ^{2} \alpha\right)$, sendo $\mathrm{MV}$ a magnitude do vetor no eixo de $90^{\circ}$, $\mathrm{m}$ o valor do astigmatismo em dioptrias e $\alpha$ o meridiano do astigmatismo em graus. A equação $\operatorname{MV}(135)=m\left(\operatorname{sen}^{2}(\alpha-45)-\cos ^{2}(\alpha-45)\right)$, calcula a diferença entre o componente de dioptrias projetado no meridiano de $135^{\circ}$ e no de $45^{\circ}$. Utilizamos ainda o EE (equivalente esférico) obtido na soma do valor esférico mais metade do valor do astigmatismo. A unidade dos valores polares é dioptria. Os resultados das análises polares vetoriais podem ser transformados novamente em valores descritivos de dioptrias esféricas, dioptrias cilíndricas e eixo.

Para exemplificar se tomarmos a refração estática clínica de $-0,25 \mathrm{DE}=-0,75 \mathrm{DC}$ no eixo de $120^{\circ}$, aplicando-se as equações de transformação teremos os valores 0,20 (MV 90), 0,32 (MV 135) e -0,63 (EE). Realizando o mesmo cálculo para uma refração por análise de frentes de onda de $-0,25 \mathrm{DE}=-1,00 \mathrm{DC}$ no eixo de $119^{\circ}$, teremos 0,25 (MV 90), 0,44 (MV135) e-0,75 (EE). Para acharmos a diferença entre as refrações subtraímos os valores [0, 20, 0, 32, $-0,63]-[0,25,0,44,-0,75]$ obtendo [-0, 05, -0, 12, 0,12], agora fazemos a conversão para a forma descritiva de refração para estes valores obtendo $0,25 \mathrm{DE}=-0,26 \mathrm{DC}$ no eixo de $34^{\circ}$ sendo esta a forma utilizada para avaliarmos as diferenças.

Para as análises descritivas, os dados foram expressos como mínimo, máximo, média, desvio-padrão ou variância a depender da variável considerada.

A estatística inferencial constituiu-se de análises univariadas, bivariadas e trivariada. Em virtude da dependência interocular entre os dados de um mesmo paciente foram feitas análises incluindo-se os dados de um olho e dos dois olhos de um paciente separadamente. A seleção do olho a ser analisado nestes pacientes fez-se de maneira aleatória pela geração, por computador, de números para sorteio. Para pacientes que possuíam dados refracionais apenas de um dos olhos, estes é que foram considerados para a análise. Nas análises univariadas, foram executados testes para todos os olhos e também para apenas um olho por paciente. Nos testes onde foram utilizados os dados de todos os olhos, a estimativa do erro padrão foi ajustada de forma robusta para acomodar a ausência de independência entre os dados de ambos os olhos de um mesmo paciente.

As análises univariadas, bivariadas e trivariadas foram executadas a partir das diferenças entre a refratometria estática clínica e a automatizada obtida por um sensor de frentes de onda. A normalidade da distribuição dos dados foi conferida através do teste de Shapiro-Wilk. As análises univariadas 
constituíram-se no estudo separado de cada um dos componentes vetoriais (EE e dos vetores de força, $1 / 2$ MV90 e $1 / 2$ MV135), verificando se as médias dos mesmos eram diferentes de zero. Para este tipo de análise foi empregado o teste t de Student. Para a análise bivariada foram estudados de forma agrupada os componentes $1 / 2$ MV90 e 1/2 MV135 verificando se a média agrupada destes valores era diferente de zero. Para a análise trivariada foram estudados de forma agrupada as diferenças das refrações do EE e dos vetores 1/2 MV90 e 1/2 MV135 verificando também se os valores eram diferentes de zero. Tanto para a análise bivariada quanto trivariada foi empregado o teste de Hotelling.

A construção dos gráficos para análises univariadas, bivariadas e trivariadas, levou sempre em conta os valores transformados das refrações (MV90, MV135 e EE).

Para a avaliação se a média das diferenças das refratometrias do EE diferia entre os tipos de ametropia (miopia, hipermetropia, astigmatismo miópico, astigmatismo hipermetrópico e astigmatismo misto) foi empregado o teste de análise de variância. Para comparações múltiplas aos pares do EE foi realizado o teste de Tukey ajustado para o número desigual de observações por grupo. Para verificar se as médias dos componentes $1 / 2$ MV90 e $1 / 2$ MV135 diferiam entre os tipos de astigmatismo (miópico, hipermetrópico e misto) foi realizado o teste de variância. Para os testes de análise de variância a normalidade da distribuição dos dados foi conferida pelo teste de Shapiro-Wilk e homogeneidade de variância foi conferida pelo teste de Levene.

Foram calculados coeficientes de correlação intraclasse dos componentes vetoriais das refratometrias clínicas e das automatizadas obtidas por um sensor de frentes de onda.

O nível de significância estatístico adotado para os testes empregados no estudo foi $\mathrm{p}<0,05$.

\section{RESULTADOS}

Os resultados da refração clinica e automatizada foram avaliados para um olho de cada paciente $(n=147)$ e para os dois olhos do paciente $(\mathrm{n}=279)$.

A tabela 1 demonstra a diferença entre as refrações clínica e automatizada, nos seus valores (vetores) para um olho de cada paciente.

Transformados os valores vetoriais para a forma convencional de escrita de uma refração, encontrou-se uma média da diferença entre as refratometrias estáticas clínica e pelo sensor de frentes de onda de -0,19 DE combinado com -0,06
DC no eixo de $15^{\circ}$, com uma variância total $(0,075+0,017+$ 0,013 ) igual a 0,105 , sendo o EE responsável por $71,4 \%$ da variância total. Estes dados são referentes à análise de um olho por paciente.

Realizou-se os mesmos cálculos para todos os olhos da amostra encontrando -0,17 DE combinado com -0,05 DC no eixo de $3^{\circ}$ com uma variância total $(0,089+0,017+0,015)$ igual a 0,021 sendo o EE responsável por $73,6 \%$ da variância total.

Para quantificar a importância do astigmatismo na diferença entre a refração clínica e automatizada, realizou-se a análise bivariada com a construção de um gráfico, onde cada ponto representa a diferença entre as refrações nos seus vetores. Quanto mais próximo das coordenadas zero, menor é a influência do astigmatismo nas diferenças entre as refrações. Por cálculos matemáticos construiu-se duas elipses onde suas abrangências dependem da variabilidade das diferenças refracionais (Gráfico 1).

Para uma avaliação incluindo os dados do EE com os vetores MV(90) e MV(135) do astigmatismo, na influência destes dados na diferença entre a refração clínica e a automatizada realizou-se uma análise trivariada (Tabela 2).

Esta análise permite criar um gráfico de dispersão tridimensional com a distribuição dos dados de uma forma espacial, demonstrando a influência do EE, e dos vetores MV(90) e MV (135), na diferença da refração clínica e automatizada, quanto mais próximo do ponto verde menor é a influência (Gráfico 2).

Realizou-se a análise de variância da diferença encontrada nas refrações clínica e a automatizada em cada tipo de ametropia, para um olho de cada paciente (Tabela 3)

A visualização da média das diferenças entre a refração clínica subjetiva e a automatizada, erro padrão e desvio padrão, nas diferentes ametropias, para um olho de cada paciente (Gráfico 3), permite observar que os valores em dioptrias destas diferenças na média são inferiores a 0,25 D, excluindose os valores para miopia (-0,339 D).

Realizou-se a análise dos valores de p para comparações múltiplas da diferença encontrada, nas refrações clínica e automatizada obtida por um sensor de frentes de onda, das ametropias, para um olho de cada paciente, utilizando o teste de Tukey (Tabela 4).

Com os dados refracionais podemos montar gráficos polares para mostrar a distribuição dos valores dos astigmatismos obtidos tanto para a refração clínica como a automatizada. Nos gráficos 4 e 5 os resultados são para um olho de cada paciente. Observar a distribuição dos valores onde no gráfico da refração clínica os valores são frações de 0,25 , enquanto que no gráfico da refração automatizada são frações de 0,01 .

\begin{tabular}{|llcccrrr|}
\hline \multicolumn{7}{|c|}{ Tabela 1. Análise univariada da diferença entre as refratometrias estática clínica subjetiva e automatizada obtida por um sensor de frentes } \\
de onda, para um olho de cada paciente
\end{tabular}




\begin{tabular}{|c|c|c|c|}
\hline Valores & EE & $1 / 2 \operatorname{MV}(90)$ & $1 / 2 M V(135)$ \\
\hline 0,078 & 0,978 & 0,208 & $-0,027$ \\
\hline 0,015 & $-0,161$ & 0,825 & 0,541 \\
\hline 0,012 & 0,135 & $-0,525$ & 0,840 \\
\hline \multicolumn{4}{|c|}{ Teste de Hotelling; $T^{2}=99,253 ; F_{3,144}=33,631 ; p<0,001$} \\
\hline
\end{tabular}

Tabela 3. Análise da variância nas refratometrias, para um olho de cada paciente. Valores em dioptrias

\begin{tabular}{|c|c|c|c|c|c|}
\hline \multirow[b]{2}{*}{ Miopia } & \multirow{2}{*}{$\begin{array}{c}n \\
42\end{array}$} & \multirow{2}{*}{$\begin{array}{l}\text { Média } \\
-0,339\end{array}$} & \multirow{2}{*}{$\begin{array}{c}\text { DP } \\
0,193\end{array}$} & \multicolumn{2}{|c|}{ IC $95 \%$} \\
\hline & & & & $-0,401$ & $-0,277$ \\
\hline A M & 88 & $-0,206$ & 0,283 & $-0,266$ & $-0,146$ \\
\hline $\mathrm{A} \mathrm{H}$ & 10 & 0,008 & 0,292 & $-0,201$ & 0,216 \\
\hline A Misto & 6 & 0,008 & 0,220 & $-0,223$ & 0,238 \\
\hline Hipermetropia & 3 & $-0,137$ & 0,223 & $-0,690$ & 0,417 \\
\hline $\begin{array}{l}A=\text { astigmatismo; } \\
F_{4,142}=5,336 ; p<0\end{array}$ & ico; & $=$ hiperme & śpico & & \\
\hline
\end{tabular}

\begin{tabular}{|c|c|c|c|c|c|}
\hline & Miopia & A M & A H & A Misto & Hipermetropia \\
\hline Miopia & $x$ & 0,148 & 0,023 & 0,139 & 0,874 \\
\hline A M & 0,148 & $\mathrm{x}$ & 0,347 & 0,608 & 0,997 \\
\hline $\mathrm{A} \mathrm{H}$ & 0,023 & 0,347 & $\mathrm{x}$ & 1,000 & 0,960 \\
\hline A Misto & 0,139 & 0,608 & 1,000 & $\mathrm{x}$ & 0,960 \\
\hline Hipermetropia & 0,874 & 0,997 & 0,960 & 0,960 & $\mathrm{x}$ \\
\hline \multicolumn{6}{|c|}{$\mathrm{A}=$ astigmatismo; $\mathrm{M}=$ miópico; $\mathrm{H}=$ hipermetrópico } \\
\hline
\end{tabular}

\section{DISCUSSÃO}

A realização do exame de aberrometria por análise de frentes de onda requer uma atenção especial na captura dos dados, alguns fatores podem influenciar na boa execução do exame, o filme lacrimal, transparência dos meios oculares entre outras causas faz com que toda avaliação deva ser estudada em separado e só confirmar o resultado se houver uma captura dos dados adequada ${ }^{(3)}$.

O sistema LadarWave utilizado neste trabalho, usa o método de Hartmann-Schack para a captura das frentes de onda, mas existem outros métodos para este tipo de análise ${ }^{(4)}$.

Há discussão de alguns autores a respeito da interdependência dos dados dos olhos de um mesmo paciente, porém não existe consenso de literatura, por isso resolvemos adotar as duas análises, de um olho e dos dois olhos de cada paciente separadamente ${ }^{(5-7)}$. Acreditamos também que a maneira mais correta para a análise de dados com astigmatismo seja a decomposição vetorial, pois a diferença aqui deve levar em conta duas forças a quantidade e direção ${ }^{(8)}$. Utilizamos no nosso trabalho a análise apresentada por Naeser et al. ${ }^{(9-10)}$ pois aqui conseguimos uma melhor identificação com as nossas idéias de avaliação para astigmatismo.

Uma dificuldade encontrada na comparação dos resulta-

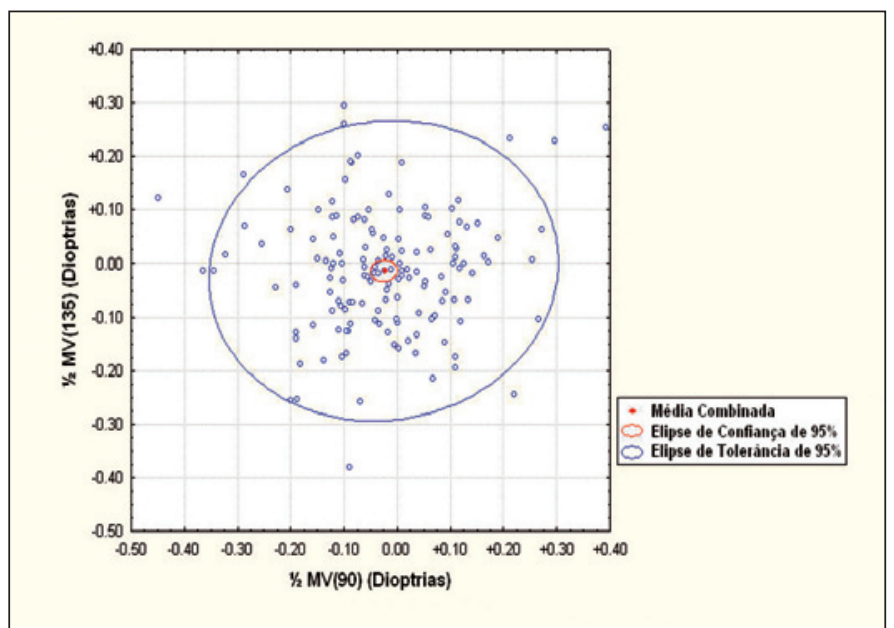

Gráfico 1 - Dados referentes à análise bivariada da influência do astigmatismo na diferenca da refração clínica e automatizada (pontos azuis) dentro de uma elipse de confiança e uma elipse de tolerância

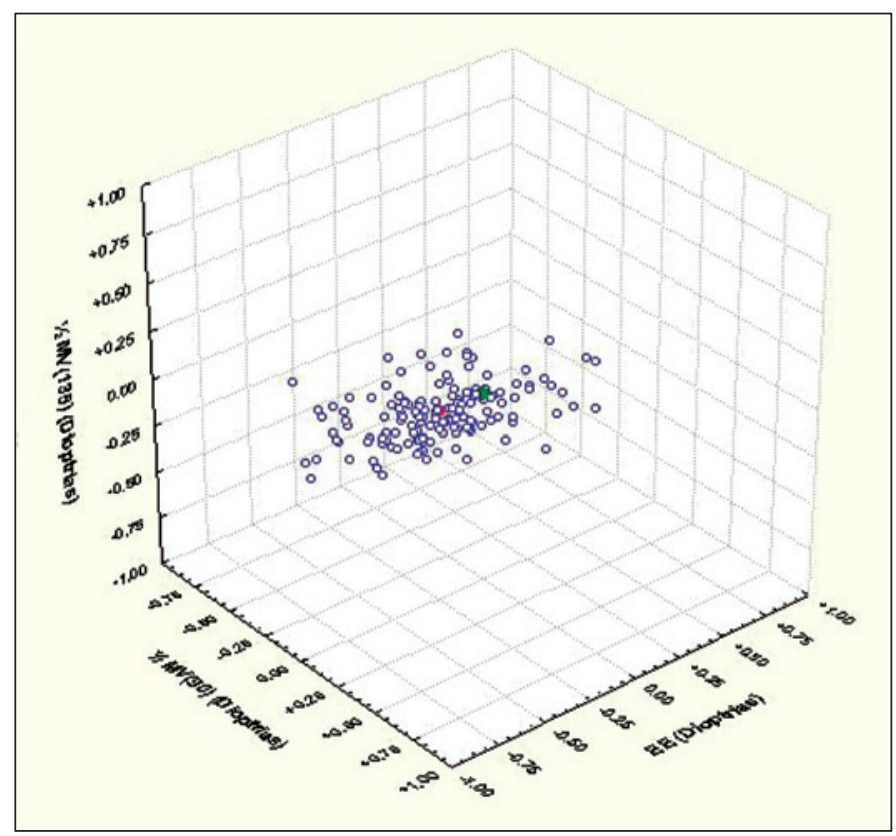

Gráfico 2 - Análise trivariada de correlação das diferenças entre as médias das refratometrias estáticas clínica subjetiva e a automatizada obtida por um sensor de frentes de onda para o EE e os vetores de astigmatismo $1 / 2 \mathrm{MV}(90)$ e $1 / 2 \mathrm{MV}(135)$ (pontos azuis). 0 ponto vermelho corresponde à média dos pontos azuis e o ponto verde seria a origem do gráfico correspondendo as coordenadas $(0,0$ e 0$)$.

dos é a magnitude dos valores refracionais obtidos pelo sensor de frentes de onda, dada com frações de $0,01 \mathrm{D}$ e na refração clínica frações de 0,25 D. Por estes dados não encontramos nenhuma refração esférica simples nas obtidas pelo sensor de frentes de onda.

A nossa análise demonstrou diferenças numéricas entre as refrações clínica e automatizadas sob cicloplegia, porém acreditamos que estas diferenças não tenham um valor clínico significativo, tanto no valor esférico menor do que 0,20 D, 


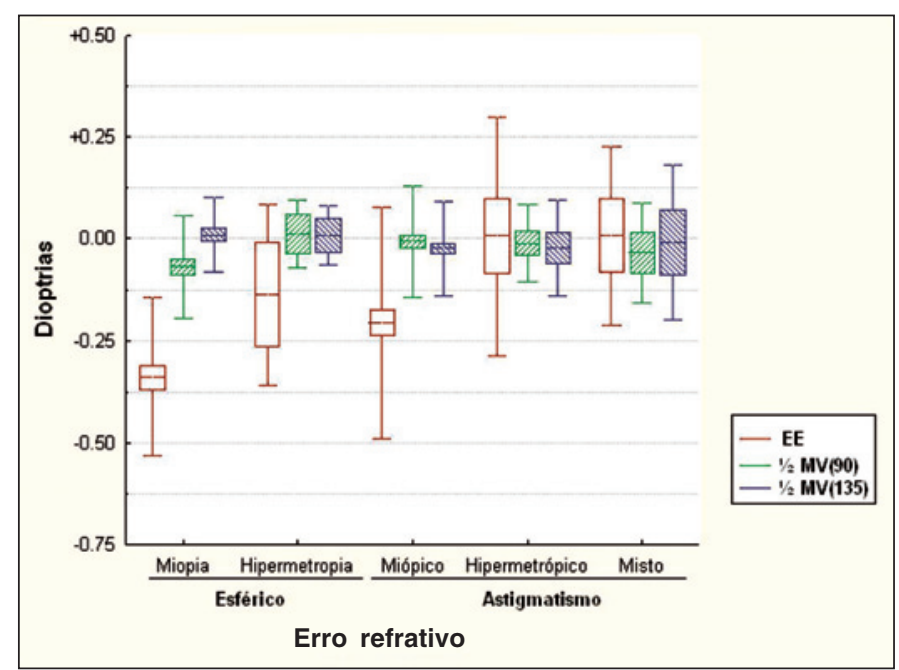

Gráfico 3 - Dados das variâncias dos valores das diferentes ametropias na diferença da refração clínica e automatizada obtida por um sensor de frentes de onda, para um olho de cada paciente, sendo a linha horizontal dentro da caixa a média, a caixa o erro padrão e a linha vertical o desvio padrão.

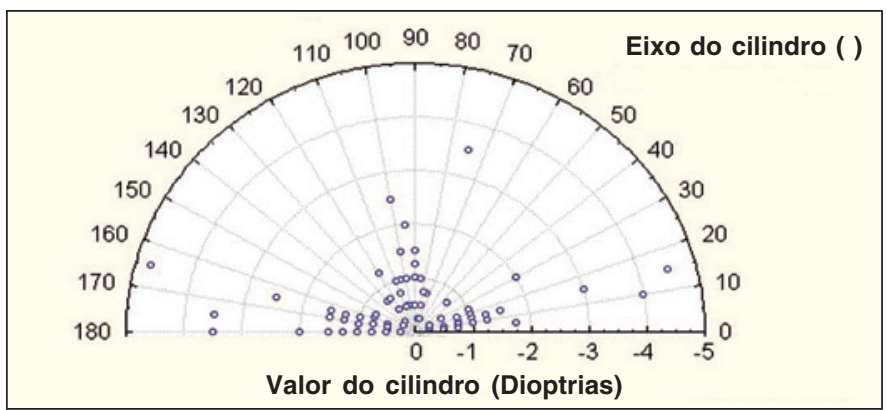

Gráfico 4 - Gráfico polar da distribuição dos valores do astigmatismo obtido da refração clínica, para um olho de cada paciente

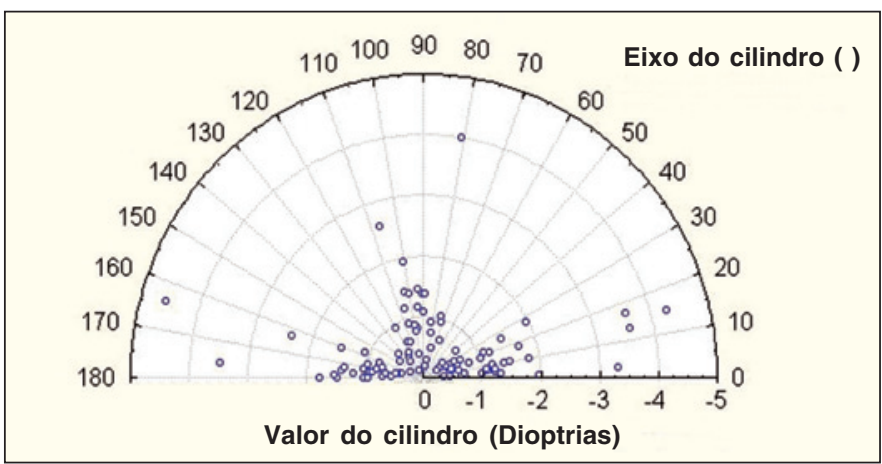

Gráfico 5 - Gráfico polar da distribuição dos valores do astigmatismo obtido da refração automatizada, para um olho de cada paciente

como no cilíndrico menor do que $0,10 \mathrm{D}$, isto tanto para um olho como para os dois olhos.

Estudos comparando a refração de olhos com e sem cirurgia refrativa utilizando dois métodos de análise, HartmannSchack e Tscherning, apresentaram diferenças pouco signifi- cativas entre as análises, porém ressalta que 50\% dos pacientes com cirurgia refrativa não tiveram seus dados analisados por uma captura não confiável ${ }^{(11)}$.

Fernández de Castro et al. compararam a refração clínica estática com a refração de três diferentes aparelhos LadarWave, ZyWave e WaveScan, todos utilizando o método de Hartmann-Schack, em 99 olhos referiram que os dados do LadarWave e do ZyWave foram mais reprodutíveis do que os do WaveScan e que as diferenças maiores foram encontradas nos resultados do astigmatismo ${ }^{(12)}$.

\section{CONCLUSÃO}

Podemos dizer que, pelos dados analisados e pela metodologia utilizada, o resultado da refração estática clínica subjetiva e automatizada obtida por um sensor de frentes de onda se assemelham, tornando este aparelho um bom recurso propedêutico para avaliação da refração.

\section{ABSTRACT}

Purpose: To evaluate and compare refractive errors obtained through clinical subjective and automatized wavefront refraction analyses in eyes under cycloplegia. Methods: Prospective study of 147 patients, 279 eyes, undergoing preoperative examination for refractive surgery. Clinical subjective refraction was performed first followed by wavefront refraction. Results on astigmatism obtained from refraction were decomposed in power vectors for statistical analyses. Data were first analyzed in one eye and then in both eyes. Results: The mean difference between clinical subjective refraction and automatized wavefront refraction on cycloplegic eyes was of -0.19 SD combined with $-0.06 \mathrm{CD}$ in the $15^{\circ}$ axis for data in one eye, and $-0.17 \mathrm{SD}$ combined with $-0.05 \mathrm{CD}$ in the $3^{\circ}$ axis for data in both eyes of the same patient. Conclusion: In the present study clinical subjective refraction and automatized wavefront refraction under cycloplegia had similar numerical values.

Keywords: Refractive errors/surgery; Refraction, ocular; Optometry; Diagnostic techniques, ophthalmological; Comparative study

\section{REFERÊNCIAS}

1. Chalita MR, Chamon W, Schor P, Freda R, Krueger R. Resultados de literatura em tratamentos personalizados com excimer laser. In: Alves MR, Chamon W, Nosé W, editores. Cirurgia refrativa. Rio de Janeiro: Cultura Médica; 2003. p.353-9.

2. Chalita MR, Finkenthal J, Xu M, Krueger RR. LADARWave wavefront measurement in normal eyes. J Refract Surg. 2004;20(2):132-8.

3. Montés-Micó R, Alió JL, Muñoz G, Pérez-Santoja JJ, Charman WN. Postblink changes in total and corneal ocular aberrations. Ophthalmology. 2004; 111(4):758-67.

4. Chamon W, Schor P, Castro Neto JC, Carvalho LA. Aspectos básicos da análise de frente de onda. In: Alves MR, Chamon W, Nosé W, editores. Cirurgia refrativa. Rio de Janeiro: Cultura Médica; 2003. p.51-64. 
682 Análise comparativa entre a refração clínica subjetiva e a automatizada obtida por sensor de frentes de onda

5. Katz J. Two eyes or one? The data analyst's dilemma. Ophthalmic Surg. 1988;19(8):585-9.

6. Katz J, Zeger S, Liang KY. Appropriate statistical methods to account for similarities in binary outcomes between fellow eyes. Invest Ophthalmol Vis Sci. 1994;35(5):2461-5.

7. Newcombe RG, Duff GR. Eyes or patients? Traps for the unwary in the statistical analysis of ophthalmological studies. Br J Ophthalmol. 1987;71(9):645-6.

8. Helena MC. Análise vetorial do astigmatismo. In: Alves MR, Chamon W, Nosé W, editores. Cirurgia refrativa. Rio de Janeiro: Cultura Médica; 2003. p.15-9.

9. Naeser K, Hjortdal JO. Bivariate analysis of surgically induced regular astig- matism. Mathematical analysis and graphical display. Ophthalmic Physiol Opt. 1999;19(1):50-61.

10. Naeser K, Hjortdal J. Multivariate analysis of refractive data: mathematics and statistics of spherocylinders. J Cataract Refract Surg. 2001;27(1):129-42.

11. Wang L, Wang N, Koch DD. Evaluation of refractive error measurements of the Wavescan Wavefront system and the Tracey Wavefront aberrometer. J Cataract Refract Surg. 2003;29(5):970-9.

12. Fernández de Castro LE, Sandoval HP, Al Sarraf O, Vroman DT, Solomon KD. Relationship between cycloplegic and wavefront-derived refraction. J Refract Surg. 2003;19(6):S677-81. 